\title{
Synthesis and Erosion Properties of PEG-Containing Polyanhydrides
}

\author{
Sijian Hou, Laurie K. McCauley, Peter X. Ma*
}

To tailor the erosion rate of polyanhydrides while retaining their surface erosion characteristics, new three-component polyanhydrides of sebacic acid, 1,3-bis( $p$-carboxyphenoxy)propane and poly(ethylene glycol) were synthesized. The hydrophilicity of the polymer increased and its mechanical strength decreased with increasing PEG content. Correspondingly, the erosion rate increases with increasing PEG content, whereas it decreases with increasing specimen thickness. This indicates that the incorporation of poly(ethylene glycol) into traditional twocomponent polyanhydrides retains their surface erosion properties while making the erosion rate tunable. The new polyanhydrides hold potential for drug delivery applications.

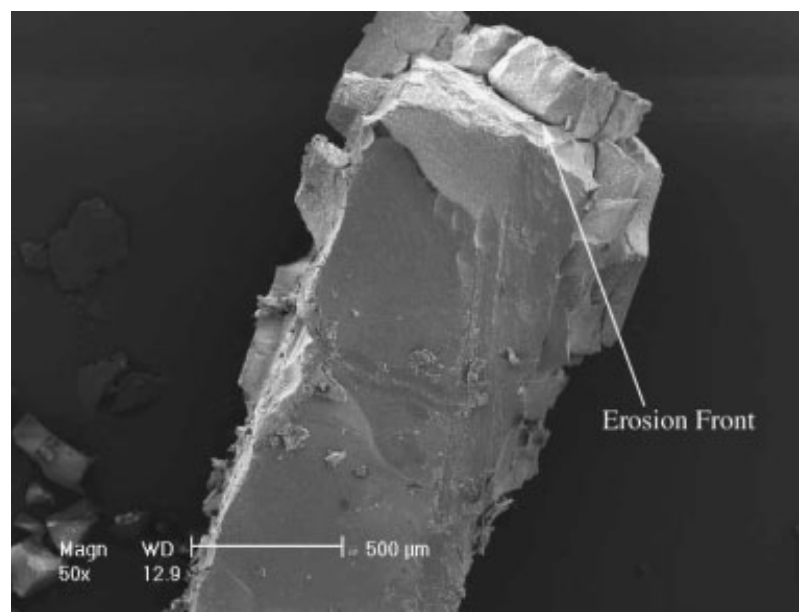

\section{Introduction}

Parathyroid hormone (PTH) is a peptide hormone that can exhibit either anabolic or catabolic actions in bone,

S. Hou, P. X. Ma

Department of Biologic and Materials Sciences, University of Michigan, Ann Arbor, MI 48109, USA

Fax: +1 734647 2110; E-mail: mapx@umich.edu

L. K. McCauley

Department of Periodontics and Oral Medicine, University of Michigan, Ann Arbor, MI 48109, USA

L. K. McCauley

Department of Pathology, University of Michigan, Ann Arbor, MI 48109, USA

P. X. Ma

Macromolecular Science and Engineering Center, University of Michigan, Ann Arbor, MI 48109, USA

P. X. Ma

Department of Biomedical Engineering, University of Michigan, Ann Arbor, MI 48109, USA depending on the dosage and delivery pattern. ${ }^{[1]} \mathrm{A}$ continuous high-dose delivery of PTH leads to a catabolic effect, while a continuous low-dose or a pulsatile highdose delivery of PTH results in an anabolic effect in bone. The high pulsatile dose is considered strongly anabolic and valuable for clinical application. Although daily injection is a feasible anabolic treatment, it is not a convenient mode of administration and is not a favorable choice of patients. Therefore controlled delivery of PTH in an anabolic fashion is highly desirable.

Biodegradable polymers have been widely pursued for controlled releases of therapeutics. ${ }^{[2-6]}$ We previously explored the possibility of using biodegradable polymers to protect the bioactivity of PTH for controlled release formulations. ${ }^{[7]}$ In that study, we demonstrated that biodegradable poly[(lactic acid)-co-(glycolic acid)] (PLGA) microspheres could be used to encapsulate PTH to protect the PTH from denaturing and to control the delivery duration through the variation of polymer structure. However, due to the bulk erosion nature of PLGA pulsatile 
release of PTH has not been achieved. The long-term goal is to fabricate PTH delivery vehicles using biodegradable polymers as the isolation and protection layer for PTH. A device having alternating polymer and PTH layers would release the PTH in a pulsatile fashion since polymer degradation and PTH release would occur intermittently.

The Langer group ${ }^{[8-12]}$ pioneered the development of polyanhydrides as surface erosion matrix materials in order to achieve zero-order release (constant release rate) of drugs uniformly distributed in the matrix. They synthesized two-component polyanhydrides of sebacic acid (SA) and 1,3-bis(p-carboxyphenoxy)propane (CPP), and investigated the degradation and erosion properties of these SA-CPP polyanhydrides. ${ }^{[13]}$ Polyanhydrides are biocompatible, biodegradable through surface erosion, and have gained increasing attention for sustained drug delivery applications. ${ }^{[14-17]}$ Such surface erosion materials hold hope for fabricating delivery vehicles to achieve a pulsatile release profile. However, the erosion of polyanhydrides is complicated and affected by many factors such as the chemical nature of anhydride bonds, crystallization of bulk polymer, and the degradation products. ${ }^{[12,18]}$ Because SA-CPP polyanhydrides are very hydrophobic, their hydrolytic degradation takes a relatively long time. These polyanhydrides erode and dissolute too slowly for the aimed pulsatile release profiles. The purpose of this work was to develop an appropriate biodegradable polymer system with tunable erosion properties to achieve the desired pulsatile release profiles. We hypothesized that a small amount of poly(ethylene glycol) (PEG) segments incorporated into the two-component polyanhydrides could retain their surface erosion characteristics, and speed up the erosion rate due to increased hydrophilicity and decreased crystallinity of the degradation products. By regulating the PEG content, the aim was to achieve polyanhydrides with adjustable surface erosion rate for the formulation of a suitable pulsatile PTH delivery device. In this work, we synthesized the new three-component polyanhydrides (SA-CPP-PEG), and studied their physical properties and erosion behavior.

\section{Experimental Part}

\section{Materials}

Sebacic acid, 4-hydroxybenzoic acid, 1,3-dibromopropane, acetic anhydride, succinic anhydride, $N, N^{\prime}$-dimethylamino-4-pyridine (DMAP), triethylamine (TEA), PEG (molecular weight: $1000 \mathrm{~g}$. $\mathrm{mol}^{-1}, \mathrm{PEG1000}$ ), poly(ethylene glycol)dicarboxy ether (molecular weight: $600 \mathrm{~g} \cdot \mathrm{mol}^{-1}, \mathrm{PEG} 600$ ), tetrahydrofuran (THF), chloroform, sodium hydroxide, and diethyl ether were obtained from Sigma-Aldrich Company. CPP was synthesized and purified in our laboratory following a published protocol. ${ }^{[9]}$

\section{Synthesis of PEG Diacid (PEG1000)}

Succinic anhydride (1.46 g, $14.6 \mathrm{mmol}$ ) and DMAP (209 mg, $1.71 \mathrm{mmol}$ ) were completely dissolved in $15 \mathrm{~mL}$ of anhydrous THF and kept at $0{ }^{\circ} \mathrm{C}$ for $30 \mathrm{~min}$. PEG (4.28 g, $\left.4.28 \mathrm{mmol}\right)$ and TEA (1.8 mL, $12.8 \mathrm{mmol}$ ) were mixed in $15 \mathrm{~mL}$ of THF and transferred slowly into the succinic anhydride solution using a syringe under the nitrogen atmosphere. The solution was stirred at $0{ }^{\circ} \mathrm{C}$ for $2 \mathrm{~h}$, and the reaction was continued at room temperature overnight.

At the end of reaction time, the reaction solution was concentrated by removing most of the solvent. The PEG diacid was precipitated in cold diethyl ether. The precipitates were dissolved in dichloromethane a second time and then reprecipitated in cold diethyl ether. The precipitates were dried under vacuum at room temperature overnight.

\section{Synthesis of Anhydride Precursors}

PEG anhydride precursors were synthesized as follows. PEG diacid (4 g, $3.3 \mathrm{mmol})$ was refluxed with acetic anhydride $(20 \mathrm{~mL}$, $210 \mathrm{mmol}$ ) for $4 \mathrm{~h}$ under nitrogen protection. The excess acetic anhydride was removed by distillation under vacuum. The crude product of PEG anhydride precursor was washed three times with diethyl ether. The precipitate was dried under vacuum at room temperature overnight. SA and CPP anhydride precursors were synthesized in our laboratory, according to the literature. ${ }^{[19]}$

\section{Synthesis of Polyanhydrides}

Anhydride precursors of SA, CPP, and PEG were charged into a predried Schlenk tube. Cycles of alternating vacuum and nitrogen purging were repeated three times, and the vacuum was maintained inside the tube at the end. The Schlenk tube was then immersed into an oil-bath and the vacuum was maintained under continuous pumping. The reaction mixture was stirred vigorously and purged with dry nitrogen for $30 \mathrm{~s}$ every $15 \mathrm{~min}$. At the end of the reaction, the resulting polymer melt was stored in a vial filled with nitrogen at $-20{ }^{\circ} \mathrm{C}$.

\section{Preparation of Disk-Shaped Specimens}

For mechanical testing and erosion characterizations, disk-shaped specimens were prepared as follows. A certain amount of a polyanhydride material was weighed and evenly dispersed in a Teflon tray ( $4 \mathrm{~cm}$ in diameter and $2 \mathrm{~mm}$ in depth). The tray was heated in a vacuum oven at $120^{\circ} \mathrm{C}$ until a transparent melt was obtained. The melt sample was then pressed using a Carver press to ensure flat surfaces. The thickness $(2,1,0.50$, or $0.25 \mathrm{~mm})$ of the polyanhydride sheet was achieved by controlling the sample weight. The disk-shaped specimens for mechanical testing and erosion characterizations $(3.55 \mathrm{~mm}$ in diameter and varying thicknesses) were obtained by punching the above-prepared polyanhydride sheets using a puncher with a diameter of $3.55 \mathrm{~mm}$ and cooling down to room temperature in a desiccator under lab-bench vacuum. The final specimen thickness was measured using a caliper accurate to $0.01 \mathrm{~mm}$. 


\section{Structural Characterization}

NMR spectra were recorded at room temperature using a Varian 300 NMR spectrometer with the residual normal solvent in the deuterated solvent as the reference.

Compressive modulus was determined at room temperature using MTS Synergie 200 mechanical tester (MTS Systems Corporation, Eden Prairie, MN). The disk-shaped specimens with dimensions of $3.55 \mathrm{~mm}$ in diameter and $2 \mathrm{~mm}$ in thickness were tested for mechanical properties. The crosshead speed was $0.5 \mathrm{~mm} \cdot \mathrm{min}^{-1}$ and the compressive modulus was defined as the initial linear modulus.

The molecular weights were calculated using standard intrinsic viscosity ( $\eta$ ) measurement. ${ }^{[20]}$ Viscosity measurement was conducted at $23^{\circ} \mathrm{C}$ in an Ubbelohde viscometer. Afflux times of polyanhydrides' solution were measured at five concentrations using anhydrous chloroform as the solvent. These afflux times were used to determine the relative viscosities and the $(\eta)$ intrinsic viscosity.

Scanning electron microscopy (Philips XL300 FEG SEM) was used to observe the morphology of degrading polymer specimens with an accelerating voltage of $10 \mathrm{kV}$. The specimens were coated with gold for $130 \mathrm{~s}$ using a sputter coater (Deak II Denton vacuum Inc.). During the coating, the gas pressure was kept at 50 mtorr and the current was $40 \mathrm{~mA}$.

\section{Erosion}

The erosion experiments were conducted in a $0.1 \mathrm{~m}$ phosphate buffer solution (PBS) at $37{ }^{\circ} \mathrm{C}$. Disk-shaped specimens of polyanhydrides were $3.55 \mathrm{~mm}$ in diameter and 1, 0.50, and $0.25 \mathrm{~mm}$ in thickness. The specimens were weighed before they were immersed into PBS. The buffer solution was renewed every $24 \mathrm{~h}$. The specimens were removed at designated times and dried in a vacuum oven at room temperature overnight. The specimens were weighed again to obtain the mass loss from the erosion. The $\mathrm{pH}$ values of the solutions were determined at room temperature using a pH meter (ORION Model 420A) calibrated with three standard solutions. For both $\mathrm{pH}$ and weight measurements, three specimens were used for each data point. The means and standard deviations were determined.

\section{Results and Discussion}

\section{Synthesis of SA-CPP-PEG Three-Component Polyanhydrides}

Two types of PEGs with molecular weights of 600 and 1000 (designated as PEG600 and PEG1000) were selected to synthesize SA-CPP-PEG polyanhydrides. The PEG600 had two carboxyl end groups while the PEG1000 had two hydroxyl end groups. As shown in Scheme 1, both hydroxyl end groups of the PEG1000 were first transformed into carboxylic groups before polymerization. This reaction was carried out under mild conditions at room temperature through the catalysis of DMAP. The corresponding PEG
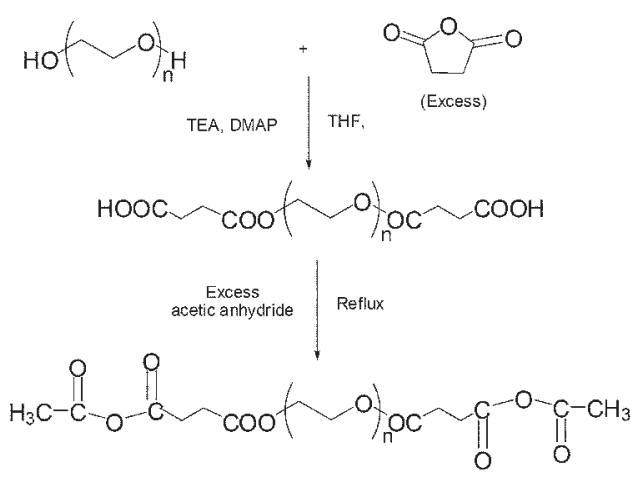

PEGIK precursor

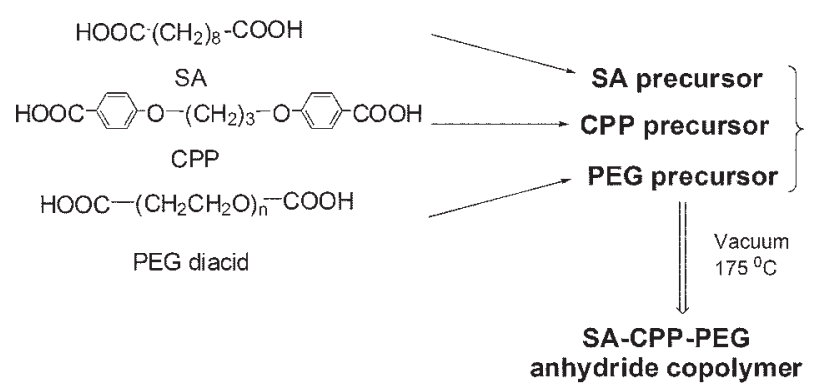

Scheme 1. Synthesis of PEG anhydride precursor and SA-CPP-PEG polyanhydrides.

anhydride precursors were obtained using similar procedures to those of other anhydride precursors (such as SA anhydride precursor) described earlier.

To focus on the effects of PEG in the anhydride polymer, the ratio between SA and CPP in polyanhydrides was kept at 80:20 and the feeding PEG was varied from 1 to $10 \%$ with respect to the total molar amounts of SA and CPP. Figure 1 shows the NMR spectrum of a typical SA-CPP-PEG polyanhydride. The peaks corresponding to PEG (3.4$3.8 \mathrm{ppm}), \mathrm{CPP}$ (6.9 and $8.0 \mathrm{ppm}$ ), and SA (1.4-1.8 ppm) confirmed the three components in the newly synthesized polyanhydrides. The PEG content in polyanhydrides was obtained by calculating the ratio of integrations of the corresponding NMR peaks.

The reaction conditions were optimized by varying reaction temperature and reaction time. As shown in Figure 2, the highest molecular weight product was prepared at $175^{\circ} \mathrm{C}$ for $3 \mathrm{~h}$. During the reaction, the viscosity of the reaction mixture gradually increased and the color of the melt changed from white to light yellow. The polymerization yield ranged from 72 to $84 \%$.

As shown in Table 1, the contents of PEG in the final products are quite close to the feeding percentages, which indicate that the PEG precursors have similar reactivity to SA and CPP precursors. Therefore, these three components are likely arranged with random sequences in the polyanhydrides. 


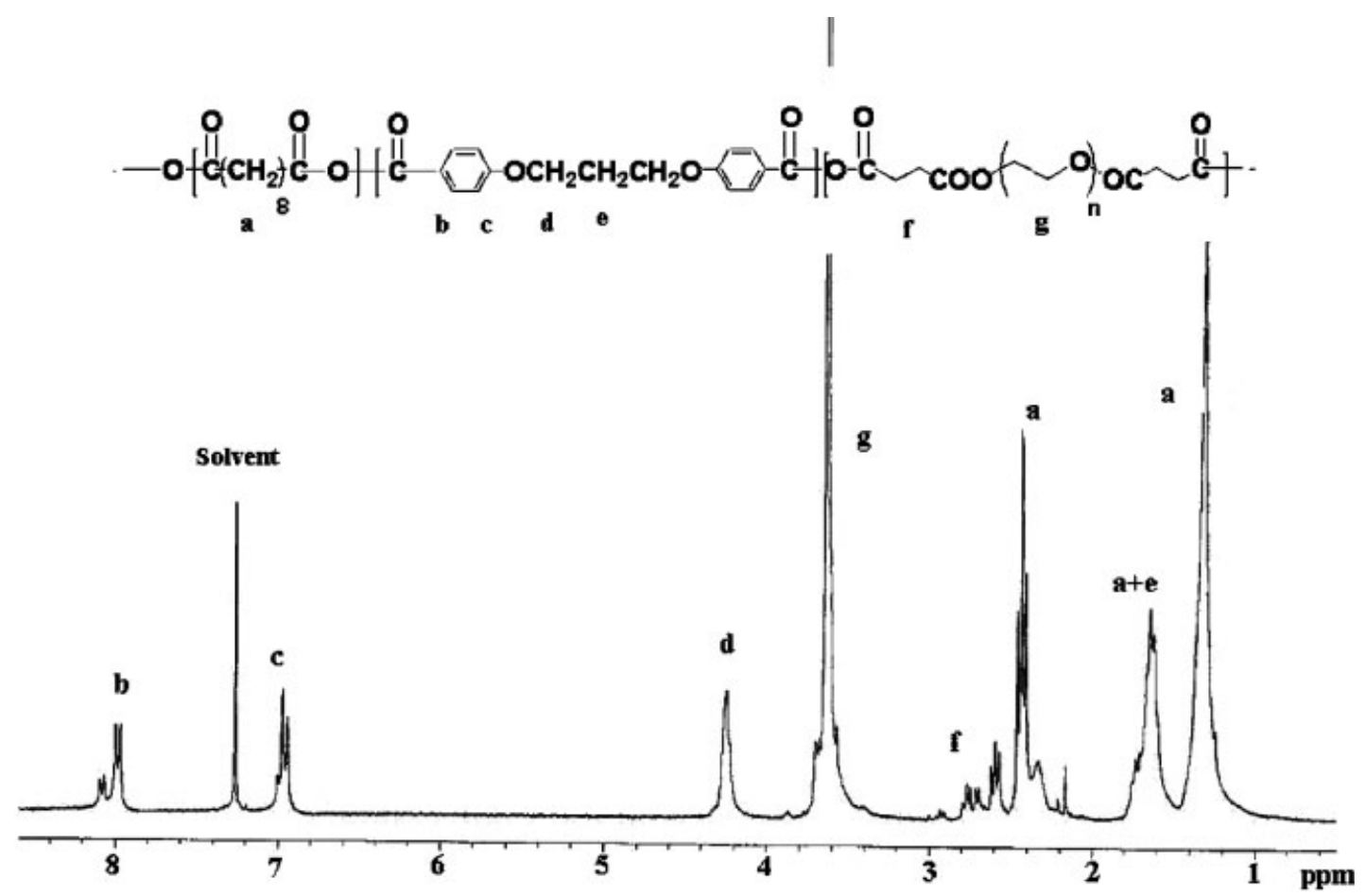

Figure 1. ${ }^{1} \mathrm{H}$ NMR spectrum of SA-CPP-PEG polyanhydrides.

Because there is a lack of relationship between the intrinsic viscosity $(\eta)$ and molecular weight $(M)$ of the new three-component polyanhydrides (containing PEG), we used the $(\eta)-M$ relationship of two-component (SA-CPP) polyanhydrides (no PEG) to estimate the molecular weights. The PEG content was low in our copolymers, so this molecular weight-intrinsic viscosity relationship allowed us to estimate the molecular weights of the three-component polyanhydrides for relative comparison purposes. At the same PEG content, the intrinsic viscosity

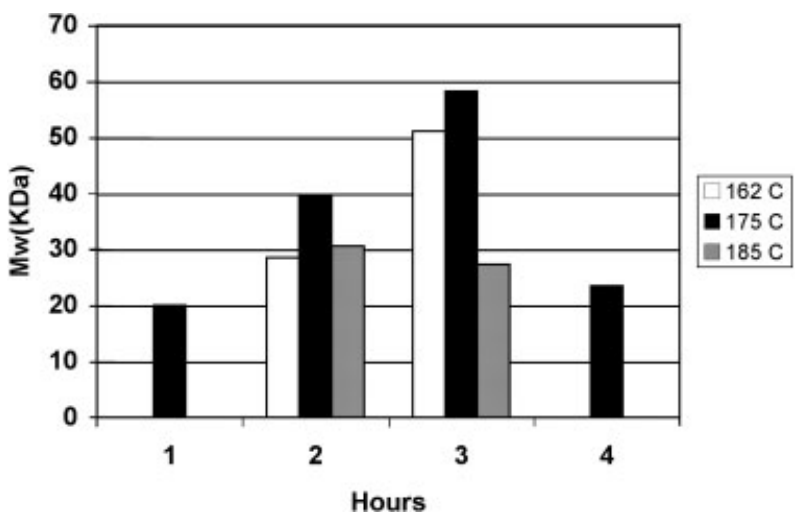

Figure 2. Effects of reaction temperature and reaction time on the molecular weight of polyanhydrides. of PEG-containing polyanhydrides with a PEG molecular weight of 600 was higher than that of polyanhydrides with a PEG molecular weight of 1000 . This could have resulted from a higher molecular weight of the PEG600containing polyanhydrides. This also could have resulted from the higher chain flexibility of the PEG1000 in a polyanhydride with a similar molecular weight to that of the PEG600-containing polyanhydride.

The results of compressive modulus and contact angle measurements are also listed in Table 1 . As the PEG content increased, the compressive modulus decreased. When the PEG content reached $10 \%$, the polyanhydrides became wax-like materials and the compressive modulus decreased drastically. The contact angle decreased as the PEG content increased, which indicated the increase in hydrophilicity. During contact angle measurement, it was noticed that the tiny drop of water gradually expanded on the polymer surface when PEG content was high $(\approx 10 \%)$, which was also a sign of increased hydrophilicity attributed to the introduction of PEG segments into the polyanhydrides.

\section{Erosion Behavior of Poly(SA-CPP-PEG)}

Erosion experiments of the synthesized polyanhydrides were conducted by measuring the mass loss of specimens 
Table 1. Properties of synthesized SA-CPP-PEG polyanhydrides.

\begin{tabular}{|c|c|c|c|c|c|c|c|c|}
\hline \multirow[t]{2}{*}{ Sample } & \multirow[t]{2}{*}{ PEG } & \multirow{2}{*}{$\frac{\mathrm{PEG}^{\mathrm{a})}}{\mathrm{mol}}$} & \multirow{2}{*}{$\frac{\mathrm{PEG}^{\mathrm{b})}}{\mathrm{mol}-\%}$} & \multirow{2}{*}{$\frac{\eta^{c)}}{d L \cdot g^{-1}}$} & \multirow{2}{*}{$\frac{\bar{M}_{\mathrm{w}}{ }^{\mathrm{d})}}{\mathrm{kg} \cdot \mathrm{mol}^{-1}}$} & \multirow{2}{*}{$\frac{\text { Yield }}{\%}$} & \multirow{2}{*}{$\frac{\text { Modulus }^{\mathrm{e})}}{\mathrm{MPa}}$} & \multirow{2}{*}{$\frac{\text { Contact angle }}{\text { degrees }}$} \\
\hline & & & & & & & & \\
\hline $\mathrm{A} 1$ & 600 & 1 & 1.1 & 0.533 & 58.8 & 72 & 58.1 & 69.3 \\
\hline A2 & 600 & 2.5 & 2.6 & 0.513 & 55.5 & 79 & 52.1 & 64.2 \\
\hline A3 & 600 & 5.0 & 5.3 & 0.385 & 35.9 & 76 & 25.6 & 51.6 \\
\hline A4 & 600 & 10.0 & 10.4 & 0.382 & 35.5 & 73 & 7.13 & 36.9 \\
\hline B1 & 1000 & 1 & 1.1 & 0.296 & 24.1 & 73 & 58.5 & 62.2 \\
\hline B2 & 1000 & 2.5 & 2.8 & 0.308 & 25.6 & 84 & 54.3 & 54.6 \\
\hline B3 & 1000 & 5.0 & 5.7 & 0.316 & 26.6 & 78 & 22.6 & 41.4 \\
\hline B4 & 1000 & 10.0 & 11.9 & 0.199 & 13.2 & 79 & 2.24 & 35.8 \\
\hline C & - & - & - & 0.497 & 52.9 & 68 & 64.2 & 89.6 \\
\hline
\end{tabular}

a) Feeding molar ratio of PEG to the sum of SA and CPP; ${ }^{b)} \mathrm{PEG}$ content in polyanhydride measured by ${ }^{1} \mathrm{H}$ NMR; ${ }^{\mathrm{c})}$ Measured in chloroform solution at $23{ }^{\circ} \mathrm{C}$; ${ }^{\text {d) }}$ Calculated based on equation $[\eta]=3.88 \times 10^{-7} \bar{M}_{\mathrm{w}}^{0.658}$ (see ref. ${ }^{[20]}$ ); ${ }^{\text {e) }}$ Compressive modulus measured at room temperature.

and $\mathrm{pH}$ value change of the PBS solutions. The disk-shaped specimens with a diameter of $3.55 \mathrm{~mm}$ and three different thicknesses $(1,0.50$, and $0.25 \mathrm{~mm})$ were prepared except for polyanhydrides containing 10\% PEG. When PEG content was $10 \%$, the polyanhydrides became very hydroscopic and too soft to prepare the specimens. During the erosion experiments, most of the specimens with a thickness of $0.25 \mathrm{~mm}$ cracked when removing them from PBS and therefore the mass loss could not be obtained.

Figure 3 shows the erosion profiles based on mass loss data. After four days of erosion, the cumulative percentage mass loss was over $80 \%$ for all the three-component polyanhydrides. Comparing the specimens with the same thickness [Figure $3(\mathrm{~A})$ or $3(\mathrm{~B})$ ], the specimens with higher PEG contents showed faster erosion rates. It should be pointed out that the sample became very fragile once the mass loss was over $80 \%$ and reached the plateau region, which was associated with the completion of erosion. The early plateau on the mass loss curve indicated that the erosion process reached completion in a short period of time. This faster erosion feature is desired for our aimed PTH delivery profiles. Two-component polyanhydrides of SA and PEG have been reported by others. ${ }^{[21]}$ Although the authors did not focus on erosion, they found that the molecular weight of the polyanhydride decreased faster as the PEG content was increased. This result was consistent with our findings.

Assuming a solid disk-shape (both specimens with or without PEG were observed to have flat surfaces under SEM), the surface areas of the specimens 1.0 and $0.5 \mathrm{~mm}$ in thickness are calculated to be 30.9 and $25.4 \mathrm{~mm}^{2}$, respectively. The corresponding volumes are 9.9 and
$4.95 \mathrm{~mm}^{3}$, respectively, which are proportional to the masses of the specimens. The surface/volume ratios for specimens 1.0 and $0.5 \mathrm{~mm}$ in thickness are 3.1 and $5.1 \mathrm{~mm}^{-1}$, respectively. For surface erosion materials, the specimens with larger surface/volume ratios should erode faster. In Figure 3(C) and 3(D), the erosion rates for the same polyanhydrides with different thicknesses are compared. For both SA-CPP and SA-CPP-PEG polyanhydrides, the thinner specimens (higher surface/volume ratio) eroded faster. The polyanhydride containing PEG (2.6\% PEG600) showed a similar mass loss behavior to that of the polyanhydride without PEG. This indicates that the polyanhydrides containing PEG retain the surface erosion properties.

It has always been a concern if the degradation byproducts (such as acids) of a biodegradable polymer result in toxicity or negatively affect biocompatibility. The SA-CPP polyanhydrides have been extensively studied for potential toxicity and biocompatibility (see review ${ }^{[22]}$ ). These in-vitro ${ }^{[23]}$ and various in-vivo ${ }^{[23-26]}$ studies indicate that the polyanhydrides are well tolerated by cells and various tissues and generally considered to be biocompatible. They are either metabolized into harmless chemicals such as carbon dioxide or excreted as lower molecular byproducts. PEG is also considered to be biocompatible, and has been extensively used in various drug delivery and tissue engineering applications (see review $^{[27]}$ ). We hypothesized that the three-component SA-CPP-PEG polyanhydrides would be biocompatible as well. To partially test this hypothesis, we examined the $\mathrm{pH}$ value change during the degradation of the threecomponent polyanhydrides in vitro. 
A)

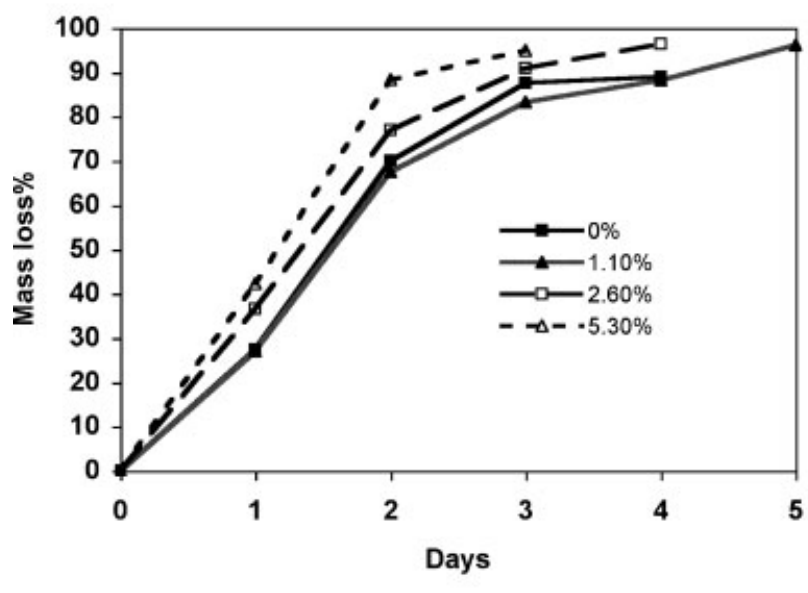

C)

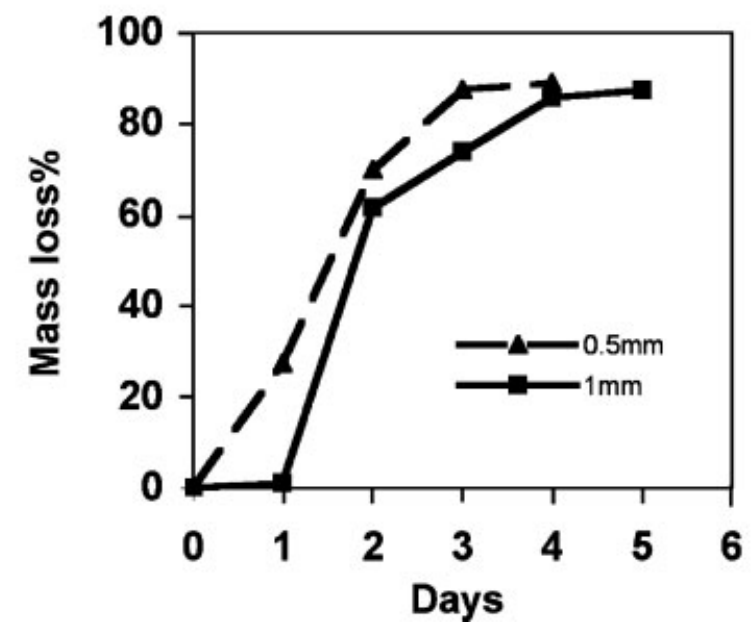

B)

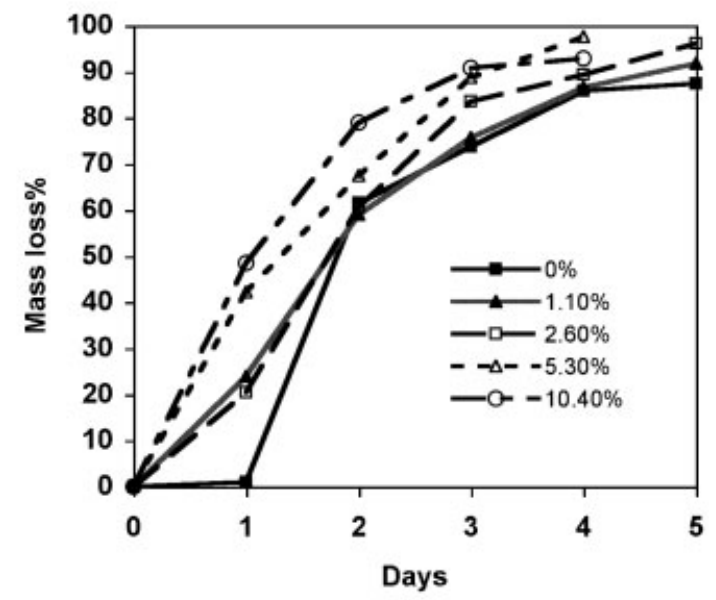

D)

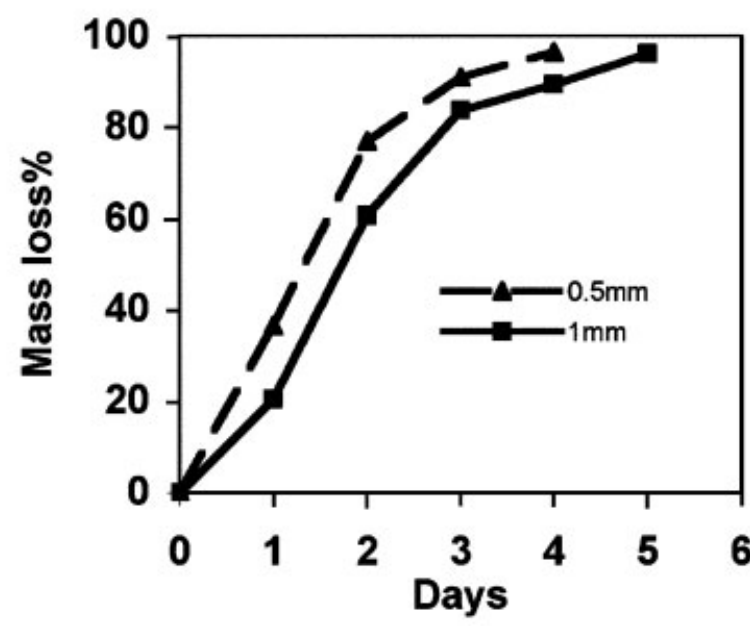

Figure 3. Mass loss profile of SA-CPP-PEG polyanhydrides: The erosion experiments were conducted at $37^{\circ} \mathrm{C}$ in $0.1 \mathrm{M}$ PBS. (A) SA-CPP-PEG600 polyanhydride with a specimen thickness of $0.5 \mathrm{~mm}$; (B) SA-CPP-PEG600 polyanhydride with a specimen thickness of $1 \mathrm{~mm}$; (C) SA-CPP polyanhydride disks with a diameter of $3.55 \mathrm{~mm}$ and two different thicknesses as indicated; and (D) SA-CPP-PEG600 (2.6\%) polyanhydride disks with a diameter of $3.55 \mathrm{~mm}$ and two different thicknesses as indicated. For each data point, three specimens were measured. The means were graphed. The standard deviations were smaller than or equivalent to the sizes of the data point symbols, and therefore were not separately marked.

The $\mathrm{pH}$ value of the PBS solutions during the erosion was always higher than 7.10 (Figure 4). The overall release of acidic byproducts from the PEG-containing polyanhydrides was not significantly different from the control SA-CPP polyanhydride, and therefore the biocompatibility is not expected to be significantly different for the SA-CPP-PEG polymers as compared to the control SA-CPP polyanhydride. The $\mathrm{pH}$ value reached the minimum after three days of erosion when the specimens were $1 \mathrm{~mm}$ thick and after two days when the specimens were $0.50 \mathrm{~mm}$ thick. This result is consistent with Figure 3, demonstrating that thinner the specimen (higher surface/ volume ratio), the faster the degradation. When samples with the same thickness were compared, higher the PEG contents, the faster the $\mathrm{pH}$ decreases during the initial degradation. The polyanhydrides with PEG content greater than $5 \%$ showed the minimum $\mathrm{pH}$ after just one day of erosion. These results demonstrate that the acceleration of erosion resulted from the increased PEG content, and that the thinner specimens degrade faster, which is the characteristic of surface erosion materials. As shown in Figure 4(C) (PEG1000 in the polyanhydrides), the minimum $\mathrm{pH}$ value was reached after three days of erosion when the PEG content was $1.1 \%$. The minimum was reached in just 
A)

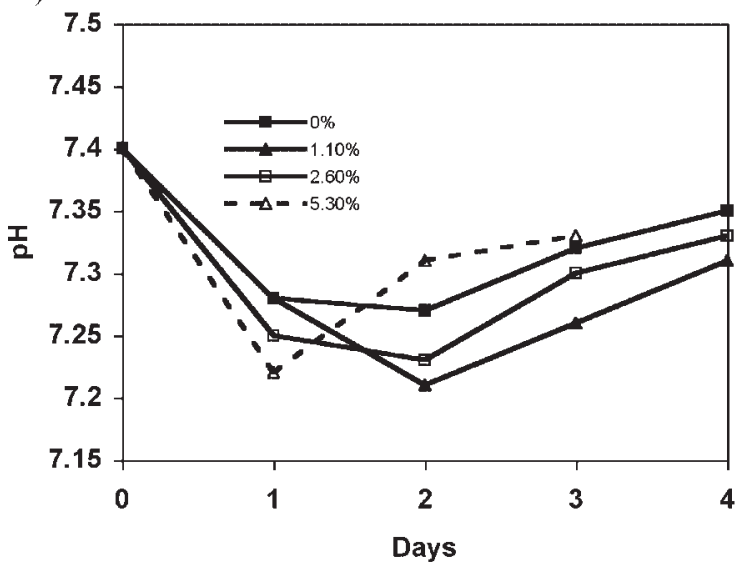

B)

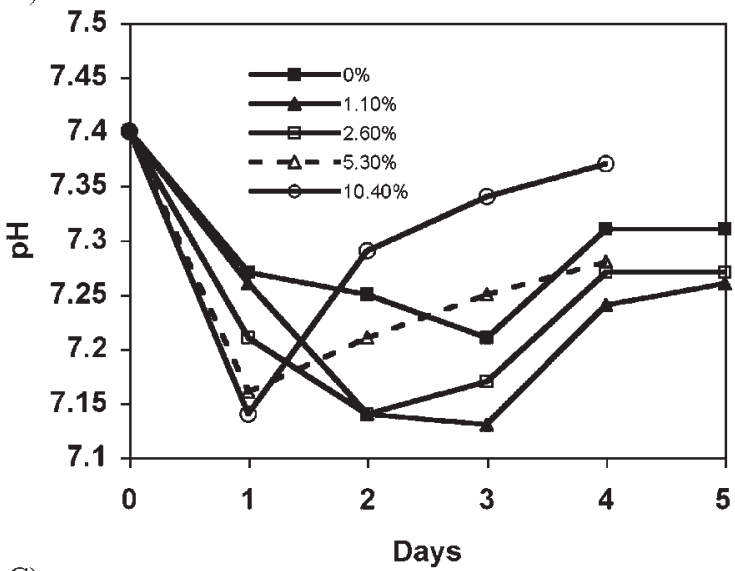

C)

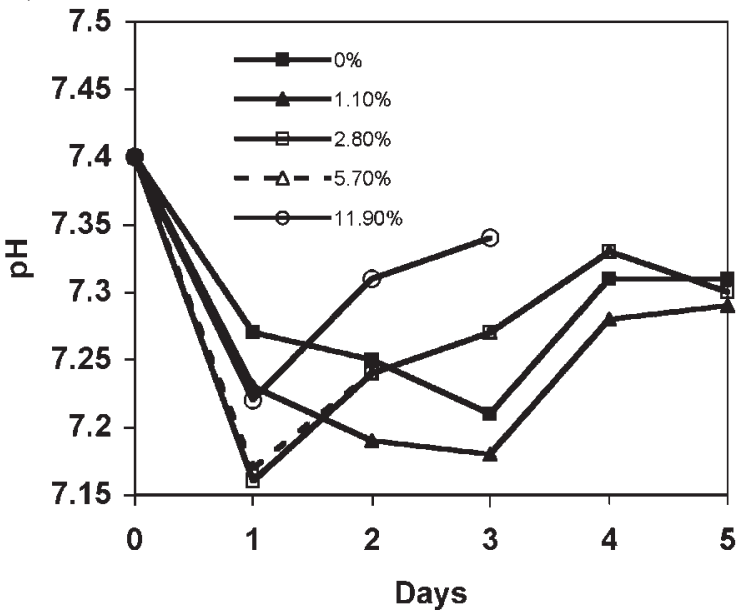

Figure 4. Change of solution $\mathrm{pH}$ value over time (erosion experiments were conducted at $37^{\circ} \mathrm{C}$ in $0.1 \mathrm{M} \mathrm{PBS}$ ). (A) SA-CPP-PEG6oo polyanhydrides ( $0.5 \mathrm{~mm}$ thick); (B) SA-CPP-PEG60o polyanhydrides (1 $\mathrm{mm}$ thick); (C) SA-CPP-PEG10oo polyanhydrides $(1 \mathrm{~mm}$ thick). For each data point, 3 specimens were measured. The means were graphed. The standard deviations were smaller than or equivalent to the sizes of the data point symbols, and therefore were not separately marked. one day of erosion when the PEG content was from 2.8 to $11.9 \%$. Considering that PEG1000 was more hydrophilic than PEG600, which was confirmed by contact angle measurements, the erosion of polyanhydrides containing PEG1000 should have been faster than that of polyanhydrides containing PEG600. This was supported by the data that polyanhydride containing 2.8\% PEG1000 reached the minimum $\mathrm{pH}$ value after one day of erosion while the polyanhydride containing a similar PEG600 content (2.6\%) reached the minimum $\mathrm{pH}$ value after two days of erosion [Figure 4(B) and 4(C)].

\section{SEM Observation}

Although mass loss data from the erosion experiments revealed surface erosion characteristics, they do not provide a direct image of surface erosion. To visualize the erosion front, SEM was used to study the morphology of the polymer materials at different stages of the erosion process. The SEM images in general are able to signify the moving erosion front, which is a typical indication of surface erosion. ${ }^{[13]}$ The porous materials formed on the surface of specimens make a very clear contrast between the eroded and noneroded parts, which allows for the estimation of the residual thickness of polyanhydrides at different erosion stages.

In Figure 5(A), there is a clear erosion front between the eroded portions and the remaining specimen. The eroded portions were so fragile that they often fell off when the SEM specimens were prepared. It was found that polyanhydride samples containing PEG exhibited faster erosion progression compared to samples without PEG. This result corroborated with those of mass loss and $\mathrm{pH}$ value measurements, indicating that the addition of PEG facilitated the erosion of polyanhydrides. The thicknesses of residual polyanhydride observed from SEM and those calculated from the remaining mass are compared in Figure 6. At higher PEG contents in the polyanhydrides (2.8\% or higher), the residual thickness was significantly smaller after erosion. The remaining thicknesses of polyanhydride specimens measured from SEM micrographs were smaller than those calculated from mass loss percentage because the portions of eroded materials still contributed to the remaining mass. The PEG-containing polyanhydride specimens became totally porous after two days of erosion with an exception of polyanhydride containing only $1.1 \%$ PEG.

\section{Conclusion}

Three-component polyanhydrides containing PEG segment can be synthesized by melting polymerization. The 
A)

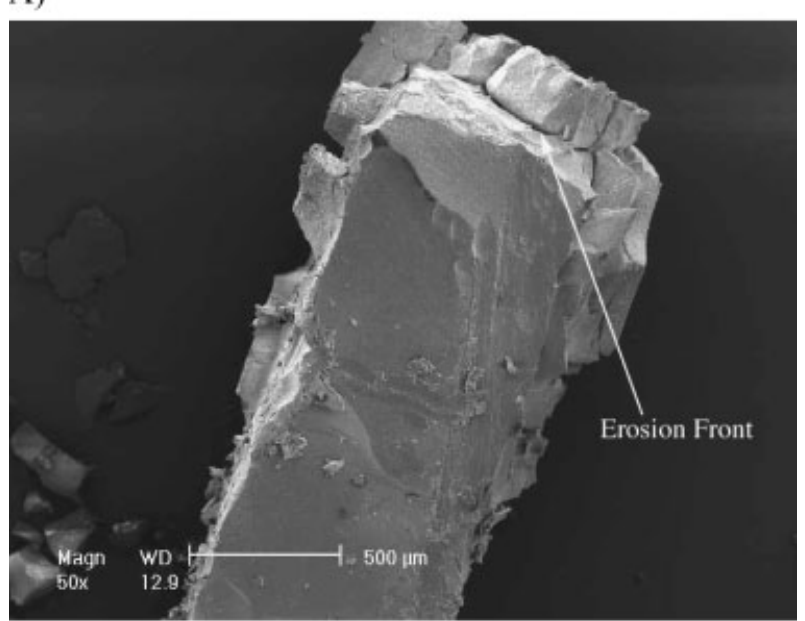

B)

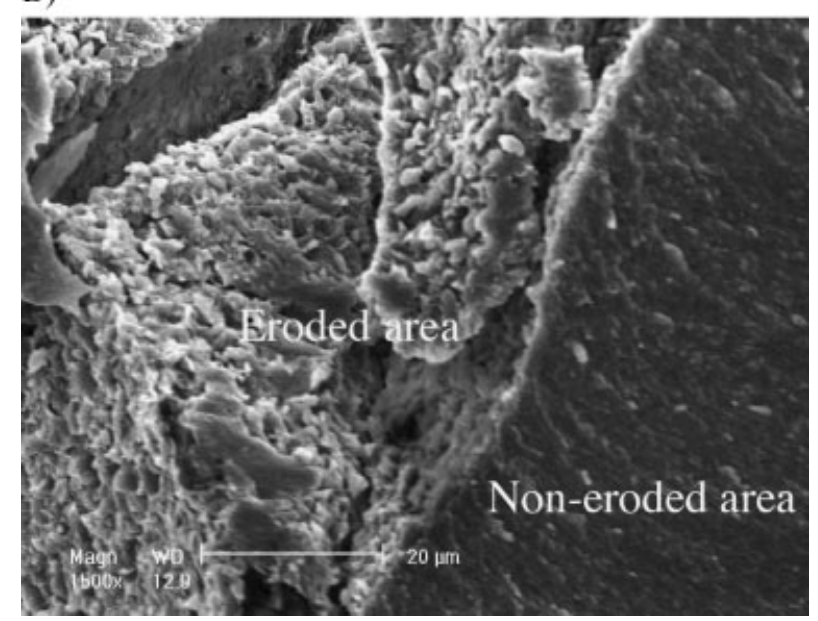

Figure 5. Cross-section of an eroding SA-CPP-PEG1000 (1.1\%) polyanhydride specimen after one day of erosion. (A) At lower magnification and (B) at a higher magnification.

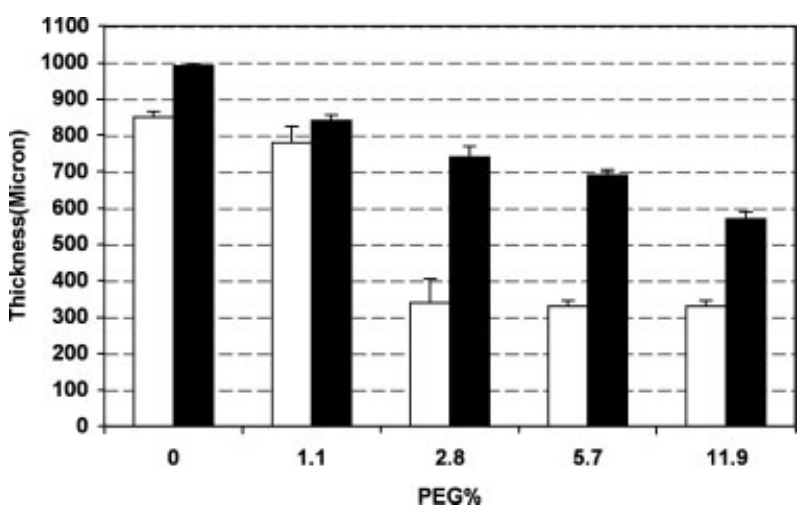

Figure 6. Thicknesses of residual polyanhydrides after one day of erosion: Open bars are the thicknesses measured using SEM, and the solid bars are calculated from the remaining mass (SA-CPP-PEG10oo polyanhydride disk specimens were $1 \mathrm{~mm}$ in thickness and $3.55 \mathrm{~mm}$ in diameter). Three specimens were measured for each data point. The means and standard deviations were graphed. surface erosion characteristics of polyanhydrides can be retained when the PEG content is low. The PEG content can be varied to tailor the erosion rate of the polyanhydrides. A higher PEG content increases the hydrophilicity and erosion rate, but decreases the elastic modulus.

Acknowledgements: We acknowledge grant support from NASA (NNC04AA21A, NASA Bioscience and Engineering Institute) and NIH (DE015384 \& DE014755: PXM; DK53904: LKM) for this work. We also thank Dr. Xiaohua Liu for his assistance in using SEM and Glenda Pettway for discussions.

Received: November 18, 2006; Revised: December 28, 2006 ; Accepted: January 10, 2007; DOI: 10.1002/mabi.200600256

Keywords: controlled release; degradation; polyanhydride; polymer; synthesis

[1] P. Morley, J. F. Whitfield, G. E. Willick, Curr. Pharm. Des. 2001, 7, 671 .

[2] J. M. Dang, K. W. Leong, Adv. Drug Deliv. Rev. 2006, 58, 487.

[3] C. Berkland, M. King, A. Cox, K. Kim, D. W. Pack, J. Controlled Release 2002, 82, 137.

[4] D. Wu, X. Zhang, C. C. Chu, J. Biomater. Sci. Polym. Ed. 2003, 14, 777.

[5] T. Yamagata, M. Morishita, N. J. Kavimandan, K. Nakamura, Y. Fukuoka, K. Takayama, N. A. Peppas, J. Controlled Release 2006, 112, 343.

[6] S. P. Schwendeman, Crit. Rev. Ther. Drug. Carrier Syst. 2002, 19, 73.

[7] G. Wei, G. J. Pettway, L. K. McCauley, P. X. Ma, Biomaterials 2004, 25, 345

[8] K. W. Leong, J. Kost, E. Mathiowitz, R. Langer, Biomaterials 1986, 7, 364

[9] H. B. Rosen, J. Chang, G. E. Wnek, R. J. Linhardt, R. Langer, Biomaterials 1983, 4, 131.

[10] H. Brem, H. C. Lawson, Cancer 1999, 86, 197

[11] K. E. Uhrich, S. M. Cannizzaro, R. S. Langer, K. M. Shakesheff, Chem. Rev. 1999, 99, 3181.

[12] A. Gopferich, J. Tessmar, Adv. Drug Deliv. Rev. 2002, 54 , 911.

[13] A. Gopferich, R. Langer, J. Polym. Sci., Part A: Polym. Chem. 1993, 31, 2445.

[14] K. Whitaker-Brothers, K. Uhrich, J. Biomed. Mater. Res. A 2006, 76,470 .

[15] D. J. Quick, K. K. Macdonald, K. S. Anseth, J. Controlled Release 2004, 97, 333

[16] F. von Burkersroda, L. Schedl, A. Gopferich, Biomaterials 2002 , $23,4221$.

[17] D. Teomim, A. J. Domb, Biomacromolecules 2001, 2, 37.

[18] J. A. Tamada, R. Langer, Proc. Natl. Acad. Sci. USA 1993, 90 552.

[19] A. J. Domb, R. Langer, J. Polym. Sci., Part A: Polym. Chem. 1987, $25,3373$. 
[20] E. Ron, E. Mathiowitz, G. Mathiowitz, A. Domb, R. Langer, Macromolecules 1991, 24, 2278.

[21] H. L. Jiang, K. J. Zhu, Polym. Int. 1999, 48, 47.

[22] D. S. Katti, S. Lakshmi, R. Langer, C. T. Laurencin, Adv. Drug. Deliv. Rev. 2002, 54, 933.

[23] K. W. Leong, P. D. D'Amore, M. Marletta, R. Langer, J. Biomed. Mater. Res. 1986, 20, 51.

[24] W. C. Domb, J. Am. Dent. Assoc. 1992, 123, 12.
[25] C. Laurencin, A. Domb, C. Morris, V. Brown, M. Chasin, R. McConnell, N. Lange, R. Langer, J. Biomed. Mater. Res. 1990, 24, 1463.

[26] R. J. Tamargo, J. I. Epstein, C. S. Reinhard, M. Chasin, H. Brem, J. Biomed. Mater. Res. 1989, 23, 253.

[27] S. J. Bryant, K. S. Anseth, "Photopolymerization of Hydrogel Scaffolds", in: Scaffolding in Tissue Engineering, P. X. Ma, J. Elisseeff, Eds., CRC Press, Boca Raton 2006, p. 71. 